\title{
Amanda Labarca Huberston: Legado y actualidad de una profesora extraordinaria ${ }^{1}$
}

\author{
Amanda Labarca Huberston: current legacy of an extraordinary teacher \\ Amanda Labarca Huberston: legado e atualidade de uma professora \\ extraordinária
}

\author{
Claudia Huaiquián Billeke ${ }^{2}$ \\ Universidad Católica de Temuco, Chile
}

Recepción: 12/03/2018

Evaluación: 05/05/2018

Aceptación: 29/06/2018

Artículo de Investigación - Reflexión

DOI: $10.19053 / 01227238.8476$

\section{RESUMEN}

El objetivo del presente artículo es reconstruir la trayectoria personal, intelectual y social de Amanda Labarca Huberston, una de las pioneras en el campo de la docencia universitaria, consagrada al magisterio de la pedagogía chilena y latinoamericana, destacada activista política y luchadora por el sufragio universal y por los derechos de las mujeres. Con el análisis teórico documental pretendo comprender la situación biográfica y la propuesta pedagógica de Amanda Labarca. El método fue cualitativo-descriptivo, con un diseño historiográfico desarrollado a partir de la triangulación metodológica entre fuentes primarias y secundarias. Los resultados pueden contribuir a promover el interés por estudiar un campo inexplorado por la investigación educativa en Chile: la historia de vida de las maestras chilenas.

Palabras clave: Revista Historia de la Educación Latinoamericana; Amanda Labarca; historia de la enseñanza; Escuela Nueva.

1 Esta investigación se enmarca en el contexto del Proyecto Fondecyt n. ${ }^{\circ} 1160409$ titulado "Historia y memoria de la escuela monocultural en la Araucanía (siglos xix y xx): dispositivos de saber-poder ejercidos por el Estado chileno hacia la sociedad mapuche".

2 Psicopedagoga. Doctora en Ciencias de la Educación. Facultad de Educación, Universidad Católica de Temuco. Correo electrónico: chuaiquian@uct.cl 


\section{ABSTRACT}

The aim of this paper is to reconstruct the personal, intellectual, and social trajectory of Amanda Labarca Huberston, one of the pioneers in the field of university teaching, devoted to the Chilean and Latin American schoolteaching. She was an outstanding political activist and fighter for universal suffrage, as well as for the rights of women. With this theoretical documentary analysis I intend to understand the biographical situation and the pedagogical proposal of Amanda Labarca. The method used was qualitative-descriptive, with a historiographic design developed with the methodological triangulation of primary and secondary sources. The results of this work may raise interest in an unexplored field of educational research in Chile: the life histories of Chilean teachers.

Keywords: Journal History of Latin American Education; Amanda Labarca; history of teaching; Escuela Nueva model.

\section{RESUMO}

O objetivo do presente artigo é reconstruir a trajetória pessoal, intelectual e social de Amanda Labarca Huberston, uma das pioneiras no campo da docência universitária, consagrada ao magistério da pedagogia chilena e latino-americana, destacada ativista política e militante pelo sufrágio universal e pelos direitos das mulheres. Fazendo uso de uma análise teórico documental, pretendo compreender a situação biográfica e a proposta pedagógica de Amanda Labarca. O método usado foi qualitativo-descritivo, com um desenho historiográfico desenvolvido a partir da triangulação metodológica entre fontes primárias e secundárias. Os resultados podem contribuir para promover o interesse para estudar um campo inexplorado pela pesquisa educacional no Chile: a história de vida das professoras chilenas.

Palavras-chave: Revista Historia da Educação Latino-americana; Amanda Labarca; história do ensino; Escola Nova.

\section{INTRODUCCIÓN}

Tened fe, y sed vosotras mismas. Templad vuestro espiritu en la ciencia, en el arte y la filosofía, para que sea consciente de vuestra fe en el triunfo del espiritu sobre todas y toda la clase de fuerzas materiales. En esa fe, uníos en grandes bandadas $y$, batiendo las alas de vuestros ensueños mejores, guiad este mundo por senderos más claros. Y sed vosotras mismas. No imitéis a nadie, y menos, al hombre. No somos iguales: somos dos seres que se complementan; dejadle a él su lote; descubrid vosotras el vuestro. (Amanda Labarca, 1927).

Amanda Pinto Sepúlveda nació en Santiago de Chile el 5 de diciembre de 1886, nueve años después de la promulgación del Decreto Amunátegui que autorizaba el ingreso de las mujeres a los liceos y a la educación superior ${ }^{4}$.

3 Amanda Labarca pronunció estas palabras en su calidad de presidenta del Consejo Nacional de Mujeres ante un grupo de estudiantes. Nadie en ese momento hubiera podido dimensionar la importancia de esta profesora y de esas ideas en la historia y en el movimiento de mujeres.

4 Luis Mardones, "Las ideas educacionales de Amanda Labarca Huberston" (tesis para optar al grado de magíster en Educación, Pontificia Universidad Católica de Chile, Santiago, 1976). 
Hija de Onofre Pinto y Sabina Sepúlveda, Amanda era la mayor de la familia. Amanda Labarca es considerada como una de las grandes educadoras de Chile e Hispanoamérica, durante la primera mitad del siglo xx. Uno de los aspectos más relevantes de los diversos aspectos de su vida y obra fue su capacidad de insertarse en un mundo abiertamente "machista" ${ }^{5}$ y hostil a las actividades femeninas ${ }^{6}$.

Desde el punto de vista profesional, alcanzó el grado de bachiller a los quince años. Entre los años 1902 y 1904 estudió en el Instituto Pedagógico de la Universidad de Chile ${ }^{7}$, donde recibió la enseñanza de eminentes profesores germanos que llegaron al país desde el año $1889^{8}$. A los 18 años obtuvo con distinción su diploma de nobleza intelectual: el título de Profesor de Estado en Castellano9. En esa fecha, 1905, se recibieron trece profesores, de los cuales solo tres fueron mujeres ${ }^{10}$. De inmediato se integró a la Asociación de Educación Nacional, la segunda organización del profesorado creada en este siglo, encontrándose con grandes pensadores y pensadoras de la educación de aquel tiempo, tales como Gabriela Mistral, Pedro Aguirre Cerda y Darío Salas ${ }^{11}$. Prosiguió sus estudios en Nueva York, en el Teachers College de la Universidad de Columbia en Estados Unidos ${ }^{12}$ y en 1912, en La Sorbonne, en París ${ }^{13}$. Fueron estos viajes los que la enfrentaron a la discusión internacional en torno a la problemática de la mujer, en un momento histórico de gran efervescencia en relación con ese tema ${ }^{14}$.

En 1915 Amanda Labarca instituyó el Club de Lectura en Santiago ${ }^{15}$, seguido de los sindicatos, asociaciones y el club social de señoras, precursoras en el discurso de los derechos femeninos y el avance del sufragio en los países del primer mundo ${ }^{16}$. Su trabajo pedagógico se inició como directora del Liceo n. 5 de Niña "Rosario Orrego" de Santiago, designación que rechazó el Partido

5 Diana Soto Arango et al., Educadores latinoamericanos y del Caribe. De la Colonia al siglo XIX (2011): 11.

6 Gonzalo Salas et al., "Amanda Labarca (1886-1975) y sus referencias psicológicas en el contexto educativo en Chile", Revista Universitas Psychologica, vol. 13, n. ${ }^{\circ} 5$ (2014): 2059-2068.

7 Allí conoció a su marido, el profesor de Historia y escritor Guillermo Labarca Huberston, de quien tomó los apellidos que la acompañaron durante toda su vida profesional. Aunque se desconocen las razones exactas de esta decisión, hay dos hipótesis: como una estrategia de posicionamiento en el campo intelectual dominado por los hombres o debido a problemas familiares.

8 Gonzalo Salas Contreras, "La influencia europea en los inicios de la historia de la psicología en Chile", Interamerican Journal of Psychology, vol. 46, n. ${ }^{\circ} 1$ (2012): 99-109.

9 Roberto Munizaga Aguirre, “Amanda Labarca Huberston", Revista Societas, vol. 1, n. 1 (1991): 307-310.

10 Emma Salas, Amanda Labarca. Dos dimensiones de la personalidad de una visionaria mujer chilena (Santiago: Ediciones Mar del Plata, 1996).

11 Felícitas Klimpel, La mujer chilena (el aporte femenino al progreso de Chile) 1910-1960 (Santiago: Editorial Andrés Bello, 1962).

12 Oportunidad que le permitió compartir con prestigiosos exponentes de la pedagogía como John Dewey y William Kirkpatrick, así como publicar el libro La educación secundaria en los Estados Unidos (1919).

13 Iván Núñez, "La historia de la enseñanza en Chile", Páginas Didácticas, vol. 9 (2002): 91-93.

14 Diamela Eltit, "Crónica del sufragio femenino en Chile" (Santiago: sernam, Servicio Nacional de la Mujer, 1994).

15 Hasta entonces las mujeres se agrupaban en torno a labores de beneficencia en organizaciones como la Cruz Roja o la Fundación Gota de Leche, que ayudaba a los más necesitados. Esta instancia de encuentro marcó un cambio radical en los movimientos femeninos.

16 Paola Mariangel Aguilar y Ana Valladares Palavecino, “Nosotras': La lucha emancipadora del sufragio femenino en Chile" (Memoria para optar al título de profesor de Educación Media en Historia y Geografía, Chillán: Escuela de Pedagogía en Historia y Geografía, 2016). 
Conservador por considerar que tenía un carácter liberal e independiente. Pese a ello, fue ratificada por el presidente Juan Luis Sanfuentes.

En 1919 fundó el Consejo Nacional de Mujeres, cuya preocupación central era la obtención de una mayor justicia social para el mundo femenino. En su calidad de presidenta, Labarca solicitó formalmente los derechos civiles y políticos.

En 1922 continuó en el campo universitario ${ }^{17}$, asumiendo como profesora extraordinaria de Psicología en el Instituto Pedagógico, de donde había egresado $^{18}$. Amanda Labarca tenía 36 años $^{19}$. En 1923 ocupó el cargo de profesora de Filosofía, sin perjuicio de los cursos de educación en los que sirvió posteriormente. Fue nombrada profesora titular de la Universidad de Chile en 1923, cargo que ejerció por más de treinta años de forma interrumpida durante la dictadura de Carlos Ibáñez del Campo. Fue el lugar donde surgieron sus textos más importantes sobre educación. Creó las Escuelas de Temporada de la Universidad de Chile.

En su extensa trayectoria académica integró el Consejo Universitario de la Universidad de Chile durante veinte años, desarrollando una labor que sobrepasó los límites nacionales al extenderse por América Latina y repercutir en el Secretariado de la Mujer en las Naciones Unidas, desde donde favoreció el progreso y la visibilidad de las mujeres puesto que la vida de las vencedoras parecía "más trágica que épica" y sus triunfos más emparentados con lágrimas y renuncias que con un futuro cargado de esperanzas y realizaciones. La educación de los sectores más pobres de la población, fue en el discurso y en la práctica, uno de los elementos más importantes del proyecto de Universidad se iba configurando en América Latina ${ }^{20}$.

En 1931 asumió como directora general de Educación Secundaria e impulsó la fundación, el 28 de marzo de 1932, del Liceo Experimental Manuel de Salas, el cual estuvo presidido por un consejo integrado, además de ella misma, por Irma Salas, Guillermo Mann, Darío Salas y Arturo Piga. Este Liceo - el primero en la enseñanza secundaria chilena en admitir a hombres y mujeres - pasó a depender de la Universidad de Chile en 1942.

17 Darío de la Fuente, “Sobre Amanda Labarca”, La Tribuna de Los Ángeles, 30 de junio de 1979, p. 3.

18 El nombramiento y la importancia del cargo fueron celebrados en un homenaje que le brindaron académicos, mujeres y estudiantes, acto al que asistió el presidente de la República, Arturo Alessandri Palma, y el entonces presidente de la Sociedad Nacional de Profesores, Pedro Aguirre Cerda.

19 Iván Núñez, "La historia..., ibíd.

20 Miguel Ángel Gutiérrez López, "Las Escuelas Normales universitarias y el problema educativo en Michoacán, 1917-1930”, Revista de Historia de la Educación Latinoamericana, vol. 18, no. 26 (2016): 137 - 156. 
Fig. 1. Amanda Labarca en su escritorio, Santiago de Chile.

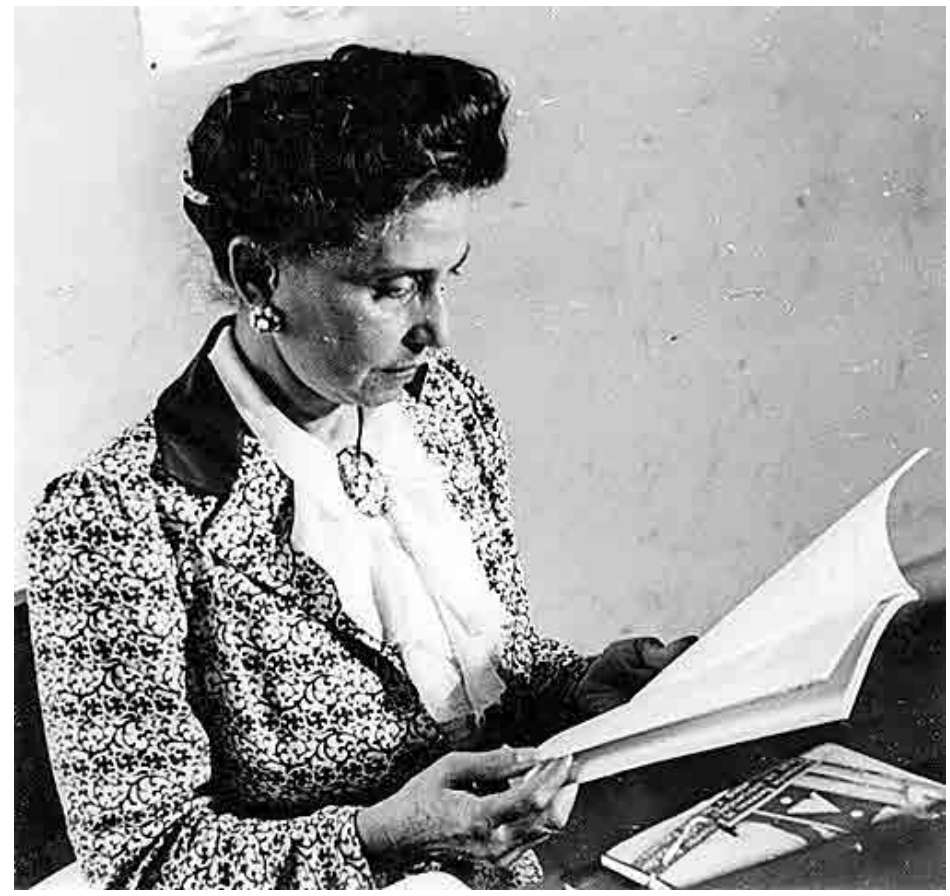

Fuente: Museo de la Educación Gabriela Mistral, Santiago de Chile, aprox. 1955

\section{Propuesta pedagógica y feminista}

La literatura sobre Amanda Labarca la ubica como una de las primeras intelectuales en generar evidencia respecto a la historia de la enseñanza en Chile. Dos libros son clave en los inicios de su carrera: Nuevas orientaciones de la enseñanza (1927), en el cual Labarca sostiene que los profesores chilenos habían empezado la adaptación de su propio sistema de educación, porque, si bien la práctica por mucho tiempo fue la de imitar teorías educacionales europeas (principalmente francesas y alemanas) y estadounidenses ${ }^{21}$, en ese momento, el movimiento de profesores - aunque muy heterogéneo - agitaba las banderas de una reforma integral de la enseñanza:

Fué [sic] al principio, un sentimiento nacionalista. Entreveíamos que nuestros problemas sociales -entre los cuales el de la educación ocupa el primer sitio- no podrán resolverse jamás con recetas copiadas de pueblos cuya evolución histórica es distinta en absoluto de la nuestra. En realidad, comenzábamos ese periodo de

21 Romina Oñate Berríos, "Ser mujer y educadora. Análisis del discurso educativo de Amanda Labarca, Olga Poblete e Irma Salas entre 1930 y 1950" (informe para optar al grado de licenciada en Historia, Universidad de Chile, Facultad de Filosofía y Humanidades, Escuela de Pregrado, Departamento de Ciencias Históricas, Santiago, 2017). 
independencia intelectual hacia lo europeo, que aún persiste, porque todavía no se ha realizado sino en mínima proporción ${ }^{22}$.

En este contexto publica un segundo libro titulado Mejoramiento de la vida campesina (1936). El texto responde a su colaboración con la redacción de un programa de enseñanza campesina (rural) presentado al Ministerio de Educación en 1934, el cual no se concretó ${ }^{23}$. No obstante, las obras donde profundiza su episteme educativa son Evolución de la Segunda Enseñanza (1938), Historia de la Enseñanza en Chile (1939) y Bases para una política educacional (1943). Se trata de tres partes de una misma serie que tienen como objetivo establecer los progresos, analizar las fallas y presentar un plan de mejoramiento de la segunda enseñanza en Chile ${ }^{24}$.

Amanda Labarca fue militante del Partido Radical y vicepresidenta de la Asociación de las Mujeres Universitarias, en 1932, y en 1933 se convirtió en una de las fundadoras del Comité Nacional pro Derechos de la Mujer ${ }^{25}$, creado junto a Elena Caffarena y otras mujeres. Desde instancias como estas promovía la lucha por la obtención del voto femenino, cuyo primer logro fue el derecho a voto en las elecciones municipales, otorgado en 1934. En 1944 fue electa presidenta de la Federación Chilena de Instituciones Femeninas (FECHIF), organización que aglutinaba a todos los movimientos y agrupaciones femeninas del país, con miras a la obtención del voto amplio para las chilenas ${ }^{26}$. La conquista del sufragio femenino tuvo lugar en 1949, año en el que se les concedió el derecho a voto para las elecciones presidenciales y parlamentarias. Participaron por primera vez en una elección presidencial en 1952, oportunidad en la que fue electo Carlos Ibáñez del Campo ${ }^{27}$.

En la línea de Historia de la Enseñanza en Chile publica Realidades y problemas de nuestra enseñanza (1953). Asimismo, la educación de la mujer propiamente como tal tiene un espacio en sus obras ¿A dónde va la mujer? (1934) y Feminismo contemporáneo (1947). En este orden de cosas, Amanda Labarca consideraba que la sociedad chilena era fruto de un mestizaje inconcluso. Pensaba que la integración racial no había logrado constituir un mestizaje social. Los sectores populares vivían en un ambiente de ausentismo material y espiritual por esta segregación social de la que fueron víctimas. Solo una educación común a todos los ciudadanos, con los mismos programas y con la base de una cultura común, podía salvar esta brecha de desintegración ${ }^{28}$.

\footnotetext{
22 Amanda Labarca, Nuevas orientaciones de la Enseñanza (Santiago [s. n.], 1927), p. 8.

23 Amanda Labarca, Mejoramiento de la vida campesina (Santiago: Edición Unión Republicana, 1936).

24 Romina Oñate Berríos, Ser mujer..., ibíd.

25 Diana Soto Arango et al., ibíd., 12.

26 Jaime Caiceo Escudero, "Amanda Labarca, Irma Salas y Mabel Condemarín, tres educadoras laicas y feministas del siglo xx en Chile", Revista de Teoria e Prática da Educação, vol. 13, n. ${ }^{\circ} 1$ (2010):105-116.

27 Ibíd.

28 Amanda Labarca, Bases para una política educacional (Buenos Aires: Editorial Losada, 1943).
} 
En materia educacional, las opciones pedagógicas de la autora estuvieron influidas por la filosofía pragmática de John Dewey y los distintos autores que constituyeron el movimiento de las Escuelas Nuevas ${ }^{29}$. Por otra parte, su experiencia como profesional de la educación y protagonista del acontecer público de la primera mitad del siglo xx, le permitió realizar un agudo análisis de la realidad nacional. Amanda Labarca contribuyó al campo de la investigación pedagógica a través de los estudios y diagnósticos profundos de la realidad educacional chilena ${ }^{30}$.

Amanda Labarca señala que no es necesario educar a los niños y niñas en internados; en todocaso, loidealsería quela familiay la escuela se complementaran, aunque ella cree que, en muchos casos, no ocurre así. El problema es que los hijos e hijas de los trabajadores de fábricas, o que trabajaban lejos de casa, son en gran medida desatendidos como consecuencia de las difíciles condiciones de vida. Incluso las sociedades civilizadas y bien acomodadas desatienden a sus hijos e hijas cuando los dejan a cargo de trabajadores domésticos. Los niños y niñas no se educan para la independencia. El internado prevé, según los estudios de la Nueva Escuela, diez horas diarias de trabajo entre las que no todo serían clases teóricas, sino que también integrarían distintas actividades, tales como viajes y proyectos $^{31}$.

Amanda Labarca tuvo una notable presencia en todo el mundo. En 1925 asumió la representación chilena en el Consejo Interamericano de Mujeres. En 1946, el presidente de la República de Chile, Gabriel González Videla, la acreditó como delegada plenipotenciaria a la Primera Asamblea de las Naciones Unidas, y en 1948 y 1949 fue jefa de la Comisión Estatus de la Mujer, en el mismo organismo internacional. Siempre estuvo ligada estrechamente a la Universidad de Chile hasta que alcanzó la edad de jubilación, en diciembre de $1955^{32}$. En 1963 fue designada Profesora Emérita de la Facultad de Filosofía y Educación, un importante grado académico solo concedido de manera excepcional. No obstante, continuó con sus actividades políticas: en 1961 creó la Liga Cívica Femenina y, en 1967, la Confederación de Organizaciones Femeninas, que presidió hasta $1970^{33}$.

En 1969 fue designada miembro de la Academia de Ciencias Políticas, Sociales y Morales del Instituto de Chile, y en 1971 fue designada directora honoraria de la Comisión Nacional Chilena de la Organización de las Naciones Unidas para la Educación, la Cultura y la Ciencia (Unesco).

29 Véase un amplio estudio sobre la aplicación de la Escuela Nueva en Latinoamérica en Revista Historia de la Educación Latinoamericana (RHELA), n. ${ }^{\circ}$ 5, 2003; Nancy Palacios Mena, "Transformación y crisis de la escuela: algunas reflexiones sobre el caso colombiano" Revista Historia de la Educación Latinoamericana, vol.15, n. ${ }^{\circ} 21$ (2013): 335-376.

30 María Teresa Rojas, "Amanda Labarca: la participación femenina en la construcción de un discurso educativo. Chile (1900-1950)", Pensamiento Educativo. Revista de Investigación Educacional Latinoamericana, vol. 34, n. ${ }^{\circ} 1$ (2011): 179-199.

31 Ana Guil Bozal y Sara Vera Gil, "Amanda Labarca, pionera de la educación y la política de las mujeres en Chile", Revista Historia de la Educación Latinoamericana (RHELA), vol. 13, n. ${ }^{\circ} 13$ (2010):143-159.

32 Si bien participó activamente en las Escuelas de Temporada de la Universidad de Chile, los inicios de su reflexión educacional se sitúan en los más de diez años en los que ocupó el cargo de directora de liceo. Tras trabajar en la Escuela Normal n. 3 de Niñas, impartió clases de castellano en el Liceo $n .^{\circ} 2$ de Niñas.

33 Jaime Caiceo Escudero, ibíd. 
A la edad de 88 años y tras largas e incansables jornadas de trabajo, Amanda Labarca falleció en Santiago el 2 de enero de 1975, dejando una huella indeleble en diversos aspectos del desarrollo educativo y social de Chile ${ }^{34}$. La Universidad de Chile en 1976 - un año después de su muerte - fundó en su memoria el premio "Amanda Labarca", destinado a reconocer los méritos de una mujer universitaria una vez por año ${ }^{35}$.

Fig. 2. Profesores de curso, Universidad de Chile. 1937

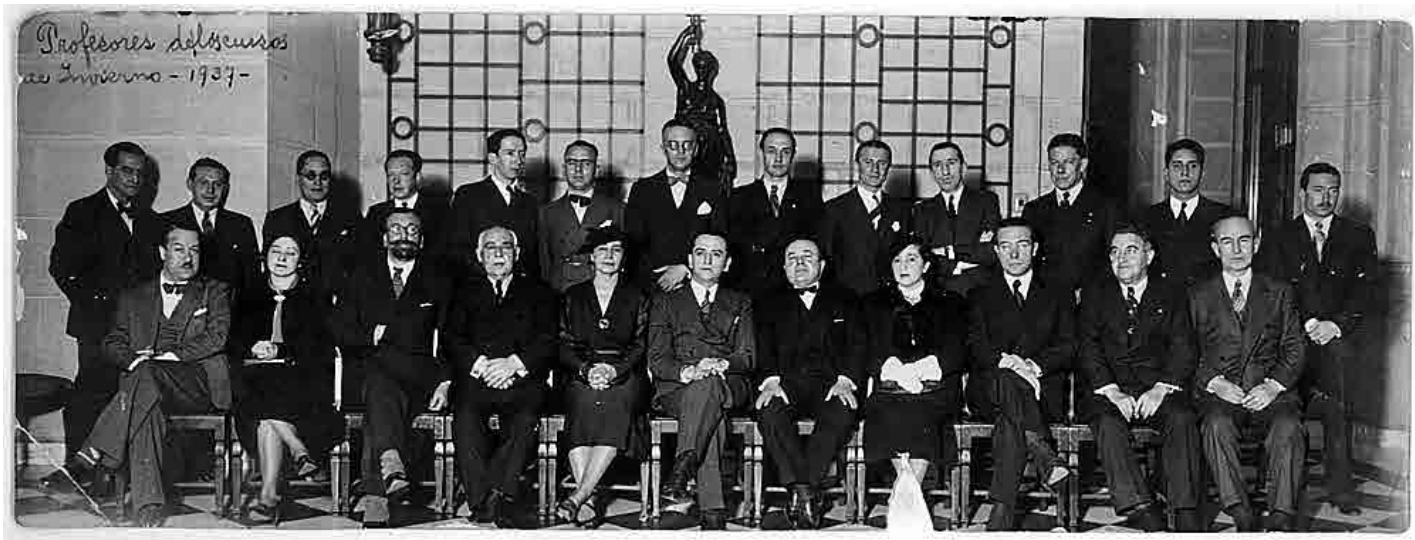

Fuente: Museo de la Educación Gabriela Mistral

\section{CONCLUSIÓN}

Es posible relevar tres grandes ideas en el transcurso de su ideario y magisterio pedagógico. La primera corresponde a la instalación en las escuelas de una nueva educación basada en el método científico. En segundo lugar, pensó la escuela como espacio social, democrático y de progreso económico para todos los estudiantes de Chile. Por último, planteó no imitar modelos de EE.UU. y Europa, sino adaptarlos a la realidad y las necesidades de una determinada población y territorio, lo que tiene relación con la idea anterior, puesto que la estructuración de la escuela debía estar condicionada según aquello ${ }^{36}$.

Otra de las críticas de Amanda Labarca se dirigió hacia la influencia del denominado "embrujo pedagógico alemán" en la pedagogía chilena, lo que implicó un desacuerdo ético político con Valentín Letelier y José Abelardo Núñez, dos próceres de la pedagogía chilena de aquel tiempo. Planteaba que el sistema jerárquico alemán profundizaba las diferencias sociales, rigidizaba las relaciones pedagógicas e inculcaba contenidos extranjerizantes, sin asidero en

\footnotetext{
34 Pamela Vargas, “Tres grandes mujeres en la historia de Chile”, Revista Arauco, 2 (2006): 28-29.

35 Jaime Caiceo Escudero, ibíd.

36 Romina Oñate Berríos, ibíd.
} 
la realidad cultural de alumnos y alumnas. La idea de no copiar para Chile los conceptos educativos europeos será una noción maestra en toda su obra ${ }^{37}$.

Amanda Labarca debe ser considerada como una de las mujeres más sobresalientes del siglo XX en Chile y América Latina. Mujer de ideales progresistas, democráticos y laicos, de tendencia radical, aspiró y ayudó al progreso de una educación para todos, así como por una sociedad igualitaria. Fue hija de la educación pública. Trabajó en la escuela, pensó en la escuela.

\section{FUENTES}

De la Fuente, Darío. “Sobre Amanda Labarca”. La Tribuna de Los Ángeles, 30 de junio de 1979, p. 3. Labarca, Amanda. Mejoramiento de la vida campesina. Santiago: Edición Unión Republicana, 1936. Labarca, Amanda. Bases para una política educacional. Buenos Aires: Editorial Losada, 1943.

Mardones, Luis. "Las ideas educacionales de Amanda Labarca Huberston". Tesis para optar al grado de magíster en Educación. Santiago: Pontificia Universidad Católica de Chile, 1976.

Oñate, Romina. "Ser mujer y educadora. Análisis del discurso educativo de Amanda Labarca, Olga Poblete e Irma Salas entre 1930 y 1950". Informe para optar al grado de licenciada en Historia. Facultad de Filosofía y Humanidades, Escuela de Pregrado, Departamento de Ciencias Históricas, Universidad de Chile, 2017.

\section{REFERENCIAS}

Caiceo, Jaime. "Amanda Labarca, Irma Salas y Mabel Condemarín, tres educadoras laicas y feministas del siglo XX en Chile". Revista de Teoría e Práctica da Educação, vol. 13, n. ${ }^{\circ}$ (2010):105-116.

Eltit, Diamela. "Crónica del sufragio femenino en Chile". Santiago: Servicio Nacional de la Mujer, 1994.

Guil, Ana y Sara Vera. "Amanda Labarca, pionera de la educación y la política de las mujeres en Chile". Revista Historia de la Educación Latinoamericana, vol. 13, n. 13 (2010):143-159.

Gutiérrez López, Miguel Ángel. “Las Escuelas Normales universitarias y el problema educativo en Michoacán, 1917-1930", Revista de Historia de la Educación Latinoamericana, vol. 18, no. 26 (2016): 137-156.

Klimpel, Felícitas. La mujer chilena (el aporte femenino al progreso de Chile) 1910-1960. Santiago: Editorial Andrés Bello, 1962.

Núñez, Iván. “La historia de la enseñanza en Chile". Páginas Didácticas, vol. 9 (2002): 91-93.

Manny-Paul, Catharine. Amanda Labarca H.: Educator to the women in Chile. (The work and writings of Amanda Labarca H. in the field of Education in Chile). Cuernavaca, México: Cuaderno 1, Cidoc, 1968.

Mariangel, Paola y Ana Valladares. "'Nosotras': La lucha emancipadora del sufragio femenino en Chile". Memoria para optar al título de profesor de Educación Media en Historia y Geografía. Chillán: Escuela de Pedagogía en Historia y Geografía, Universidad del Bío-Bío, 2016.

Munizaga, Roberto. “Amanda Labarca Huberston". Revista Societas, vol. 1, n. ${ }^{\circ} 1$ (1991): 307-310.

Palacios Mena Nancy, "Transformación y crisis de la escuela: algunas reflexiones sobre el caso colombiano" Revista Historia de la Educación Latinoamericana, vol. 15, n. 21 (2013): 335-376.

Rojas, María. "Amanda Labarca: la participación femenina en la construcción de un discurso educativo. Chile (1900-1950)". Pensamiento Educativo. Revista de Investigación Educacional Latinoamericana, vol. 34, n. ${ }^{\circ} 1$ (2011): 179-199.

37 Catharine Manny-Paul, Amanda Labarca H.: Educator to the women in Chile (The work and writings of Amanda Labarca H. in the field of Education in Chile). (Cuernavaca, México: Cuaderno 1, Cidoc, 1968). 
Salas, Gonzalo, Rodolfo Mardones, Miguel Gallegos y Fernando Ponce. “Amanda Labarca (18861975) y sus referencias psicológicas en el contexto educativo en Chile". Revista Universitas Psychologica, vol. 13, n. 5 (2014): 2059-2068.

Salas, Gonzalo. "La influencia europea en los inicios de la historia de la psicología en Chile". Interamerican Journal of Psychology, vol. 46, n. ${ }^{\circ} 1$ (2012): 99-109.

Salas, Emma. Amanda Labarca. Dos dimensiones de la personalidad de una visionaria mujer chilena. Santiago: Ediciones Mar del Plata, 1996.

Soto Arango, Diana, Jesús Paniagua, José Rubens Lima Jardilino y María Cristina Vera de Flachs, coords. Educadores latinoamericanos y del Caribe. De la Colonia al siglo xIx. Tomo III. Tunja, León, Sao Paulo, Córdoba: Colciencias; Universidad Pedagógica y Tecnológica de Colombia; Din; Rudecolombia; Universidad de León, España; Universidad Nove de Julho; Universidad Federal de Ouro Preto, Brasil; Universidad Nacional de Córdoba, Argentina; Universidad de Cuenca, Ecuador; Universidad de los Andes, Venezuela; Universidad de San Carlos de Guatemala; shela-hisula, 2011.

\section{Cómo citar:}

Huaiquián Billeke, Claudia. "Amanda Labarca Huberston:

Legado y actualidad de una profesora extraordinaria" Revista

Historia de la Educación Latinoamericana. Vol. 20 No. 31 (2018): 15-24

DOI 10.19053/01227238.6245 